\title{
Anterior lamina cribrosa surface depth in healthy Saudi females
}

\author{
This article was published in the following Dove Press journal: \\ Clinical Ophthalmology \\ I June 2017 \\ Number of times this article has been viewed
}

\author{
Amira El-Agamy $1,2, *$ \\ Fayrouz Oteaf ${ }^{\prime, *}$ \\ Mohamed Berika ${ }^{3,4}$ \\ 'Department of Optometry and Vision \\ Sciences, College of Applied Medical \\ Sciences, King Saud University, Riyadh, \\ Saudi Arabia; ${ }^{2}$ Mansoura Ophthalmic \\ Center, Faculty of Medicine, Mansoura \\ University, Mansoura, Egypt; \\ ${ }^{3}$ Rehabilitation Science Department, \\ College of Applied Medical Sciences, \\ King Saud University, Riyadh, Saudi \\ Arabia; ${ }^{4}$ Anatomy Department, Faculty \\ of Medicine, Mansoura University, \\ Mansoura, Egypt \\ *These authors contributed equally \\ to this work
}

Purpose: This study was conducted to determine normative profile of anterior lamina cribrosa surface depth (ALCSD) in healthy Saudi females using Topcon Three-Dimensional (3D) Optical Coherence Tomography (OCT) 2000 - Spectral Domain (SD-OCT). In addition, the correlation between ALCSD and other clinical factors such as age, refractive error, intraocular pressure (IOP), central corneal thickness, anterior chamber depth, axial length, retinal nerve fiber layer thickness, and disk area was also assessed.

Design: This study was a prospective, nonrandomized, cross-sectional, observational, and quantitative study.

Methods: This study included 191 eyes of 191 healthy Saudi females from the College of Applied Medical Sciences of King Saud University. Stereoscopic disk photographs were reconstructed using Topcon 3D OCT-2000 for all subjects. ALCSD was measured at three planes (superior, middle, and inferior) and defined as the distance from Bruch's membrane opening level (reference line) to anterior lamina cribrosa surface. Average of ALCSD at all planes was defined as mean ALCSD of the eye. Correlation between ALCSD and all the clinical factors was performed by linear regression analysis. Paired $t$-test was performed in order to compare ALCSD at all planes.

Results: In this study, the average ALCSD was 371.88 $\pm 114.62 \mu \mathrm{m}$ (range, 155-647.6 $\mu \mathrm{m})$. Paired $t$-test showed a significant difference between superior and middle planes $(P=0.004)$ and middle and inferior planes $(P=0.013)$. Using the same test, no significant difference between superior and inferior planes $(P=0.820)$ was observed. Generally, the largest ALCSD was in the middle plane. In addition, linear regression analysis showed no significant correlation between ALCSD and associated clinical factors.

Conclusion: This work is the first to provide the normative profile of ALCSD in Saudi females using Topcon 3D OCT-2000. Further studies are recommended for males, different ethnic populations, high myopic eyes, and different age groups using advanced imaging techniques such as enhanced depth imaging OCT.

Keywords: optical coherence tomography, anterior lamina cribrosa surface depth

\section{Introduction}

Lamina cribrosa (LC) is the primary site of axonal injury of retinal ganglion cells (RGCs) in glaucoma. It consists of collagenous beams intersecting and branching, forming a mesh-like structure in the scleral canal of optic nerve head (ONH). RGC axons and retinal blood vessels pass through its pores. ${ }^{1}$

Pathogenesis of glaucomatous cupping is composed of two stages: prelaminar and laminar. Prelaminar cupping is produced by progressive thinning and atrophy of prelaminar neurons, increasing both depth and width of the cup. Laminar cupping is formed by continued posterior bowing and excavation of LC beneath the anterior 
scleral canal. ${ }^{2}$ In chronic glaucoma, anatomical alterations in LC are supposed to induce interruption of axoplasmic flow within RGC axons that leads to sight blindness. ${ }^{3}$

Imaging instruments, such as optical coherence tomography (OCT), can help us to understand glaucomatous pathologic changes in LC by providing objective quantitative measures of LC depth. So, they can support initial glaucoma diagnosis and detect early progression. Thus, patient's treatment strategy can be modified to preclude visual deterioration. ${ }^{4}$

The purposes of this study were to determine the normative profile of anterior lamina cribrosa surface depth (ALCSD) in healthy Saudi females using Topcon ThreeDimensional (3D) OCT-2000 - Spectral Domain (SD-OCT) and to assess correlation between ALCSD and other clinical factors such as age, refractive errors, intraocular pressure (IOP), central corneal thickness (CCT), anterior chamber depth (ACD), axial length (AXL), and retinal nerve fiber layer (RNFL) thickness and disk area.

\section{Methods}

\section{Study design}

This study was a prospective, nonrandomized, cross-sectional, observational, and quantitative study. It was approved by the research ethics committee of College of Applied Medical Sciences (CAMS) of King Saud University (KSU), Riyadh, Saudi Arabia. It adhered to tenets of the Declaration of Helsinki. Written informed consent was obtained from all participants after explaining all procedures of the study.

\section{Subjects}

This study included 191 eyes (only right eye) of 191 healthy Saudi females from CAMS of KSU from 27 October 2015 to 30 November 2015 . The age range was $18-26$ years (mean $20.76 \pm 1.627$ years). Each participant underwent complete ophthalmic examinations, including medical and ocular history, visual acuity measurement, refraction, and air-puff tonometry. In addition, measurement of CCT, ACD, and AXL was performed using Pentacam HR (Oculus, Wetzlar, Germany). In addition, stereoscopic disk photography and measurement of RNFL thickness were done using Topcon 3D OCT-2000 system (SD-OCT).

The inclusion criteria were IOP $<21 \mathrm{mmHg}$ without antiglaucoma treatment and normal looking optic disk without any signs of glaucomatous or ischemic optic neuropathy.

The exclusion criteria were best-corrected visual acuity worse than $20 / 40$, spherical refraction $>+2.0$ D and $<-3.50 \mathrm{D}$, cylinder correction $>+2.0 \mathrm{D}$, amblyopic eye, or poor image quality. In addition, subjects with a history of ocular surgery, intraocular disease (eg, diabetic retinopathy or retinal vein occlusion), or neurologic disease (eg, chiasma or parachiasma lesion) that were identified to affect optic nerve structure or visual field were excluded.

\section{Stereoscopic disk photography}

Stereoscopic disk photography was obtained by Topcon 3D OCT-2000 system (SD-OCT). The imaging was achieved using a $3 \mathrm{D}$ disk analysis mode with $512 \times 128$ resolution (128 horizontal scan lines composed of 512 A-scans covering the optic disk), $6 \times 6 \mathrm{~mm}$. The images were obtained only when the quality score was $\geq 56$. 3D volumetric images were reassembled from the B-scan images, and en face images were made from the 3D images using image-processing software (FastMap ${ }^{\mathrm{TM}}$ software produced by Topcon).

All stereoscopic disk photographies were done by one trained examiner. The 3D image was reconstructed at first. Then, the three equidistant planes were made and defined as plane 1 , plane 2 , and plane 3 . In this model, plane 2 corresponded to the mid-horizontal plane, and planes 1 and 3 corresponded to the superior and inferior planes, respectively. The ALCSD was measured at all planes, defined as the distance from the line connecting the two Bruch's membrane opening (BMO) edges (reference line) to the anterior LC surface. It was measured in the direction perpendicular to the reference plane at three points: the maximum depth point and two additional points (100 and $200 \mu \mathrm{m}$ apart from the maximum depth point to temporal direction). We selected only the temporally adjacent points because the LC at the maximally depressed point was masked by the shadow of the central vessel trunk. The average of three measurements was taken as the ALCSD of each plane (Figures 1-6). The average of the ALCSDs from all planes was defined as the mean of ALCSD of the eye. ${ }^{5}$

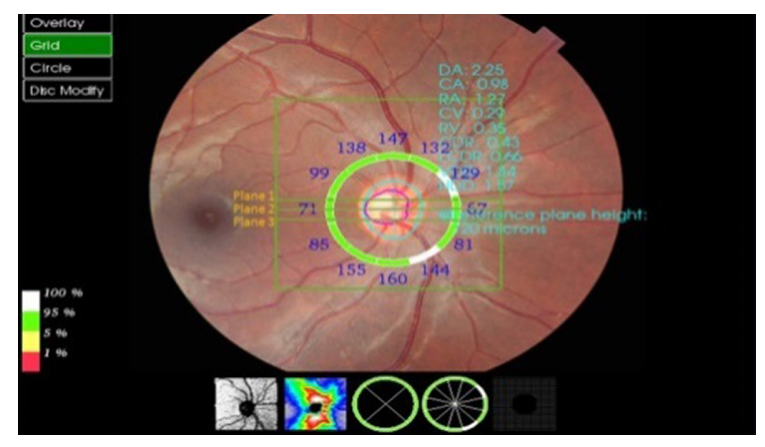

Figure I Case I: fundus photography shows three equidistant planes defined as plane I, plane 2, and plane 3.

Note: In this model, plane 2 paralleled to the mid-horizontal plane, and planes I and 3 paralleled to the superior and inferior planes, respectively. 

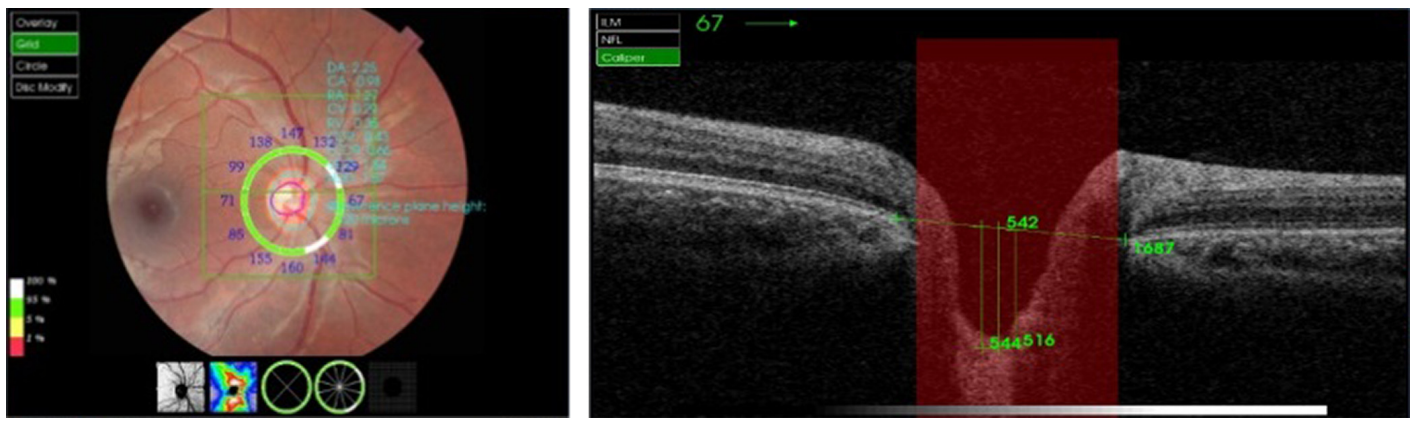

Figure 2 Case I: the average of three measurements $(542,516$, and $544 \mu \mathrm{m})$ was taken as the ALCSD $(534 \mu \mathrm{m})$ at the superior plane. Abbreviation: ALCSD, anterior lamina cribrosa surface depth.
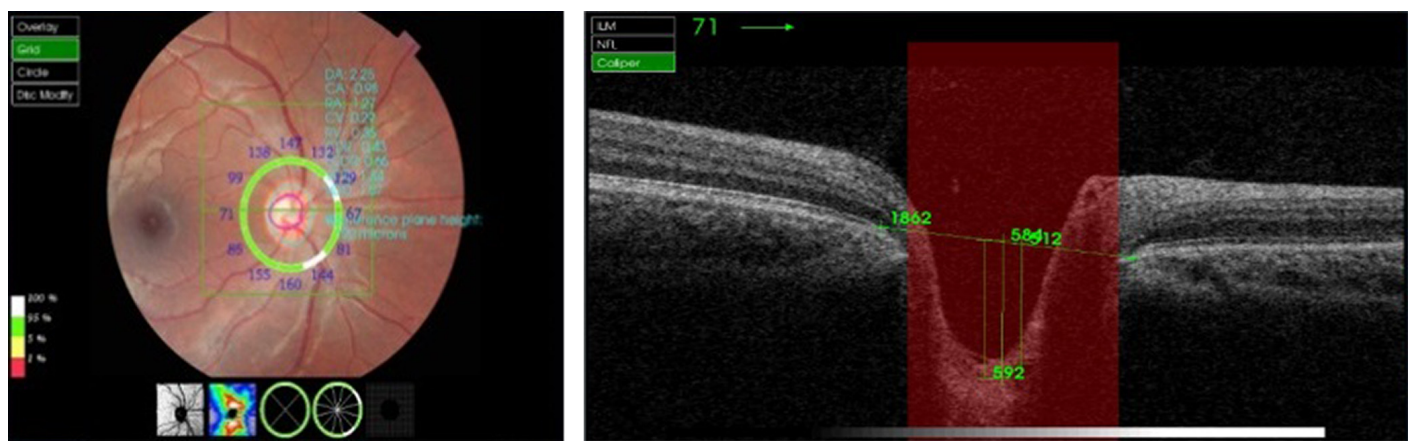

Figure 3 Case I: the average of three measurements $(412,585$, and $592 \mu \mathrm{m})$ was taken as the ALCSD $(529.6 \mu \mathrm{m})$ at the mid-horizontal plane. Abbreviation: ALCSD, anterior lamina cribrosa surface depth.
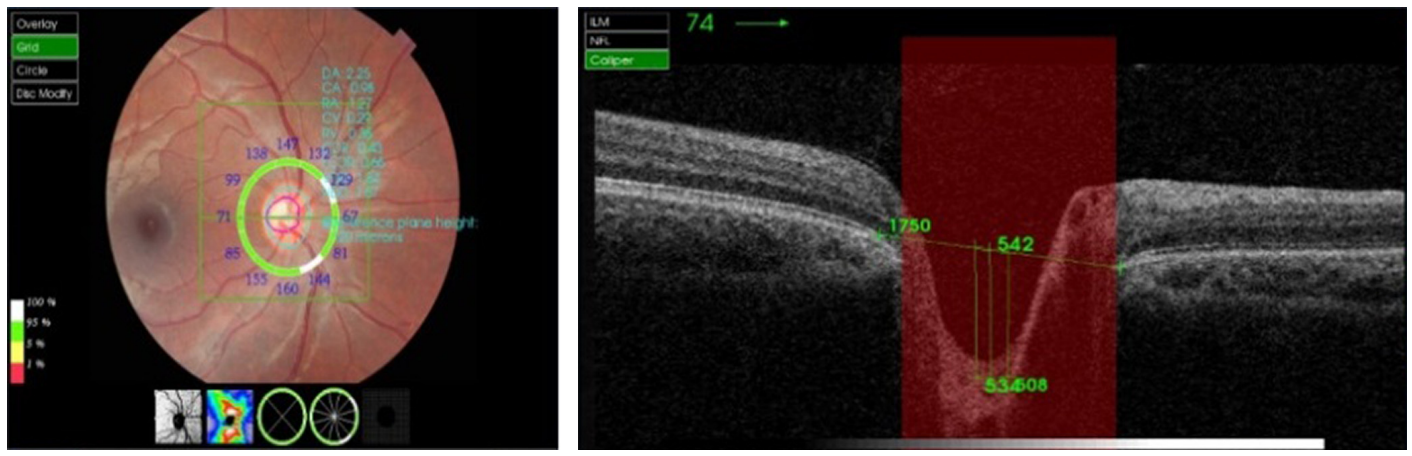

Figure 4 Case I: the average of three measurements $(542,508$, and $534 \mu \mathrm{m})$ was taken as the ALCSD $(528 \mu \mathrm{m})$ at the inferior plane. Abbreviation: ALCSD, anterior lamina cribrosa surface depth.
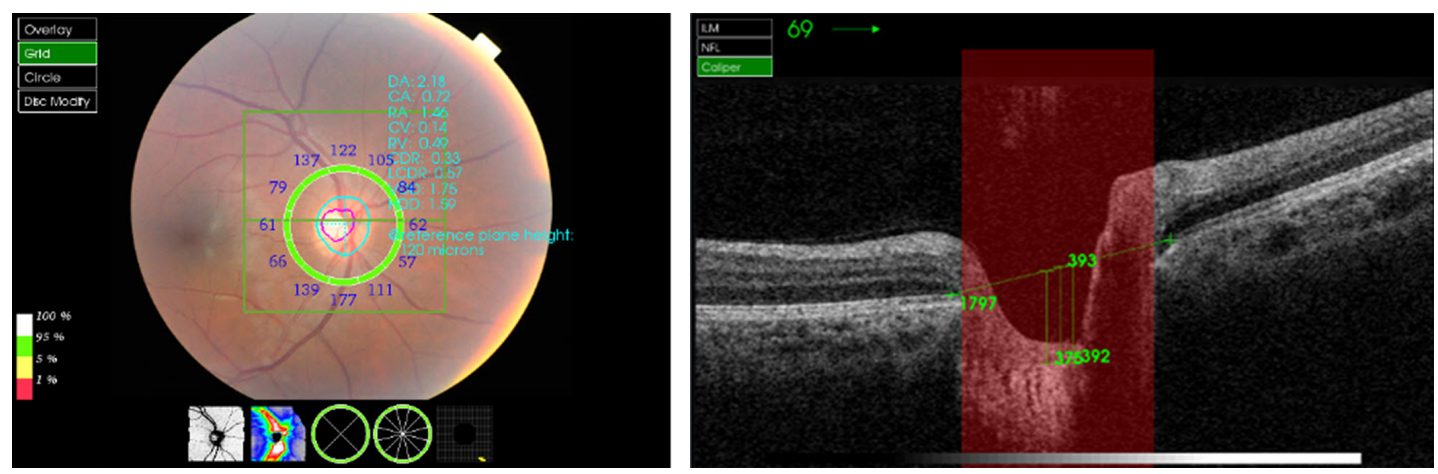

Figure 5 Case 2: the average of three measurements $(393,392$, and $375 \mu \mathrm{m})$ was taken as the ALCSD $(386.6 \mu \mathrm{m})$ at the superior plane. Abbreviation: ALCSD, anterior lamina cribrosa surface depth. 

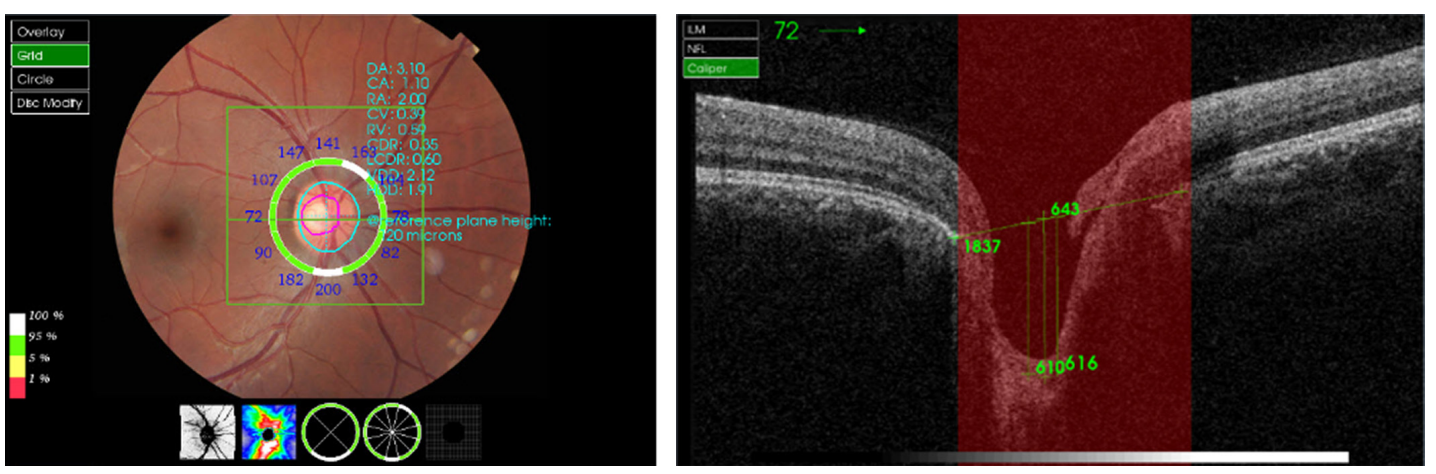

Figure 6 Case 3: the average of three measurements $(643,616$, and $610 \mu \mathrm{m})$ was taken as the ALCSD $(623 \mu \mathrm{m})$ at the inferior plane. Abbreviation: ALCSD, anterior lamina cribrosa surface depth.

\section{Statistical analysis}

Statistical analysis was performed with commercially available software (SPSS for Windows, version 22). $P<0.05$ was considered as statistically significant. Correlation between ALCSD and all clinical factors was performed by linear regression analysis. Paired $t$-test was done for comparison of ALCSD at all planes.

\section{Results}

A total of 191 eyes of 191 normal Saudi females from CAMS of KSU participated in this study. Four eyes were excluded for suspicion of optic neuropathy. In addition, 91 eyes were excluded because of poor LC visibility (Figures 7 and 8). Therefore, a total of 96 eyes were enrolled for analysis. The mean of ALCSD at superior, middle, and inferior planes was $368.08 \pm 116.04,379.86 \pm 117.12$, and $366.59 \pm 120.98 \mu \mathrm{m}$, respectively. The mean of ALCSD was $371.88 \pm 114.62 \mu \mathrm{m}$. The demographics and clinical characteristics and the mean of ALCSD are summarized in Table 1.

The mean frequency distribution of the ALCSD showed a curve ranging from 155 to $647.6 \mu \mathrm{m}$ (Figure 9). Paired $t$-test showed a significant difference between superior and middle planes $(P=0.004)$ and middle and inferior planes $(P=0.013)$ but no significant difference between superior and inferior planes $(P=0.820)$. In general, the ALCSD was largest in the middle plane (Figure 10). In addition, linear regression analysis showed no significant correlation between ALCSD and clinical factors associated with it.

\section{Discussion}

Many studies demonstrated that increased ALCSD, which may be induced by posterior displacement and/or thinning of the LC, is a basic element of glaucomatous optic neuropathy. In addition, in experimental glaucoma, early RNFL loss has been confirmed to be preceded by LC displacement. ${ }^{6,7}$ So, ALCSD could be considered as a valuable guide for predicting the development of glaucoma in suspected patients. In addition, determination of normative profile and factors associated with ALCSD variation is very useful. To our knowledge, this is the first study to assess ALCSD in healthy subjects in Saudi Arabia using Topcon 3D OCT-2000.
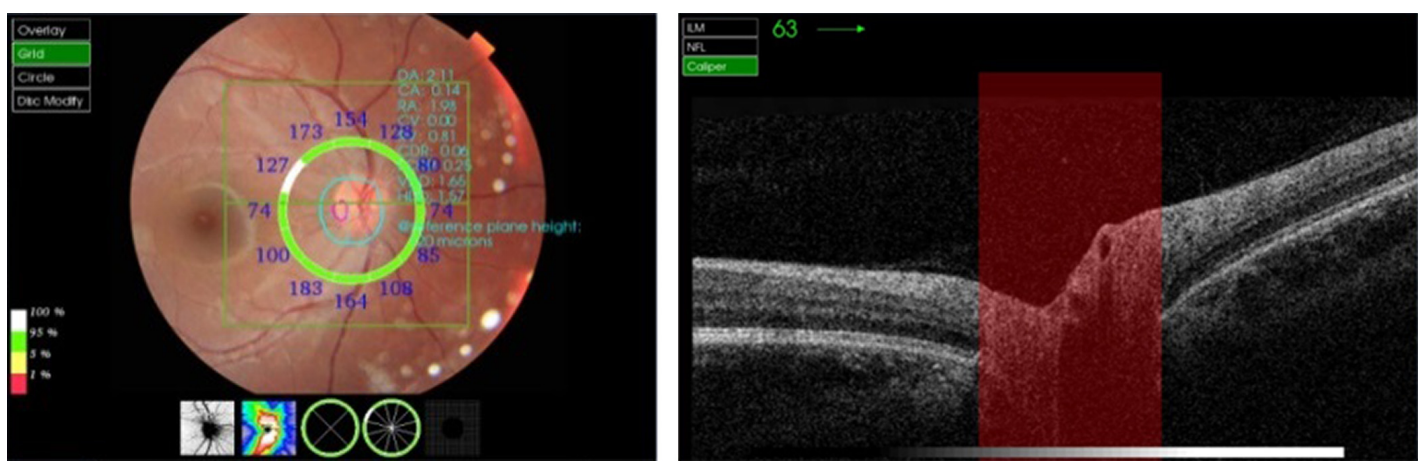

Figure 7 Case 4: poor visibility of LC due to small cup (cup/disc ratio: 0.06 ). Abbreviation: LC, lamina cribrosa. 

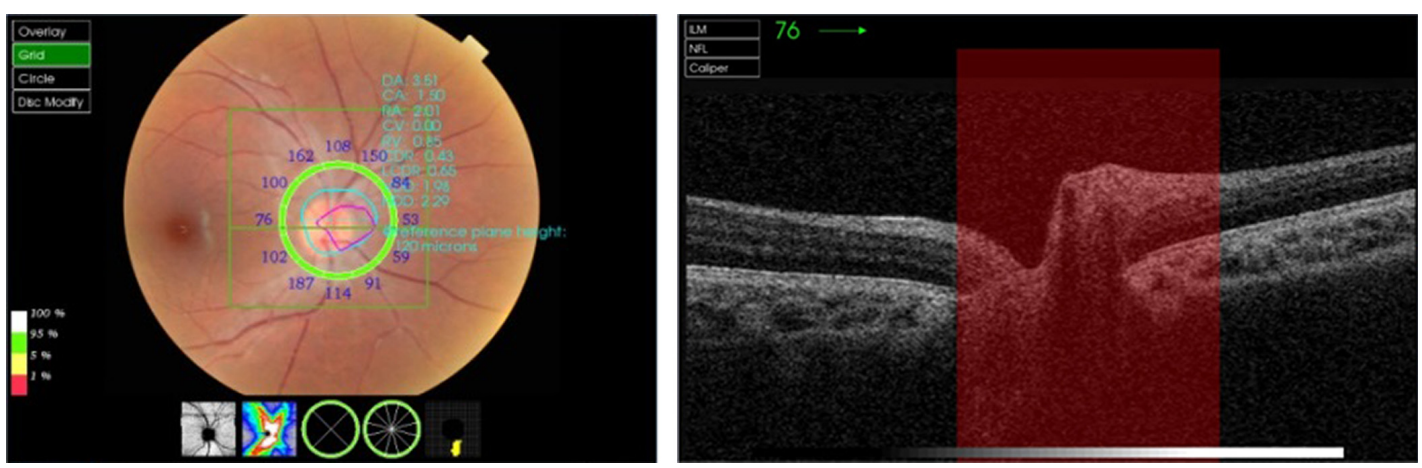

Figure 8 Case 5: poor visibility of LC (masked by the shadow of the central vessel trunk). Abbreviation: LC, lamina cribrosa.

In this study, the mean of ALCSD of healthy eyes was $371.88 \pm 114.62 \mu \mathrm{m}$. This was matched with the studies of Seo et al, ${ }^{5}$ Furlanetto et al, ${ }^{8}$ Sawada et al, ${ }^{9}$ and Park et al ${ }^{10}$ that reported the mean of ALCSD of normal eyes to be $402.06 \pm 101.46,453 \pm 81,388$, and $344 \mu \mathrm{m}$, respectively. In our study, the ALCSD was largest in the middle plane, whereas in Furlanetto et $\mathrm{al}^{8}$ and Seo et $\mathrm{al},{ }^{5}$ it was largest in the superior mid periphery.

In the present study, we reported that ALCSD was not correlated with age, refractive errors, IOP, CCT, or disk area. This was in agreement with Seo et al..$^{5}$ In addition, we found that ALCSD did not correlate with AXL. This was in disagreement with Seo et al, ${ }^{5}$ who demonstrated that the AXL had a significant negative correlation with ALCSD. This disagreement may be because our study enrolled only healthy subjects with AXL $<25 \mathrm{~mm}$ with mild myopia $(<-3.50 \mathrm{D})$,

Table I Demographic and characteristics of study subjects

\begin{tabular}{lll}
\hline Characteristics & Mean \pm SD & $\begin{array}{l}\text { Range } \\
\text { (min-max) }\end{array}$ \\
\hline Age, years & $20.76 \pm 1.627$ & $18-26$ \\
IOP at the time of disk & $17.90 \pm 2.355$ & $1 \mathrm{I}-2 \mathrm{I}$ \\
scanning, mmHg & $-0.75 \pm 1.077$ & +2.00 to -3.50 \\
Spherical equivalent, D & $554 \pm 28.507$ & $495-62 \mathrm{I}$ \\
$\mathrm{CCT}, \mu \mathrm{m}$ & $3.49 \pm 0.299$ & $2.76-4.32$ \\
$\mathrm{ACD}, \mathrm{mm}$ & $23.50 \pm 0.872$ & $21.46-25.37$ \\
AXL, mm & $113.99 \pm 14.432$ & $92-186$ \\
RNFL, $\mu \mathrm{m}$ & $2.24 \pm 0.355$ & $1.63-3.5 \mathrm{I}$ \\
Disk area, mm ${ }^{2}$ & $0.33 \pm 0.121$ & $0.12 \pm 0.52$ \\
Cup/disk ratio & $368.08 \pm 116.04$ & $149-655$ \\
Superior plane ALCSD, $\mu \mathrm{m}$ & $379.86 \pm 117.12$ & $136-663.3$ \\
Middle plane ALCSD, $\mu \mathrm{m}$ & $366.59 \pm 120.98$ & $124-652.3$ \\
Inferior plane ALCSD, $\mu \mathrm{m}$ & $371.88 \pm 114.62$ & $155-647.6$ \\
\hline Mean ALCSD, $\mu \mathrm{m}$ &
\end{tabular}

Abbreviations: SD, standard deviation; IOP, intraocular pressure; CCT, central corneal thickness; ACD, anterior chamber depth; AXL, axial length; RNFL, retinal nerve fiber layer; ALCSD, anterior lamina cribrosa surface depth. but their study included subjects with AXL $>25 \mathrm{~mm}$ (high myopic eye, $\geq-8.0 \mathrm{D}$ ). Moreover, it was supposed that during childhood while the globe grows axially, temporal sclera moves back and flattens. So, the optic nerve is drawn toward the temporal direction, leading to a tilted appearance of the optic disk. This may result in shorter LC depth. ${ }^{11}$

In addition, our study found no correlation between ALCSD and RNFL. This was in agreement with Ren et al, ${ }^{12}$ who reported the absence of a significant univariate association between ALCSD and age, IOP, or RNFL. We did not find a correlation between age and ALCSD. The explanation of this finding may be because our study enrolled only younger age group (ranging from 18 to 25 years) and no older age

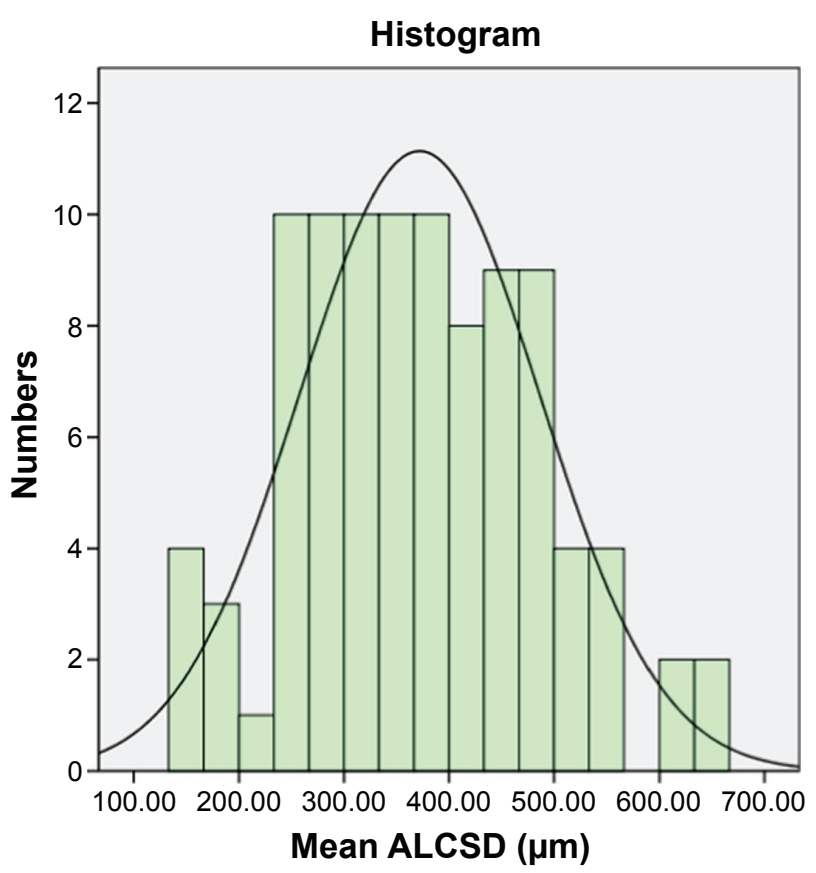

Figure 9 Histogram showing distribution of ALCSD variation among healthy subjects.

Abbreviation: ALCSD, anterior lamina cribrosa surface depth. 


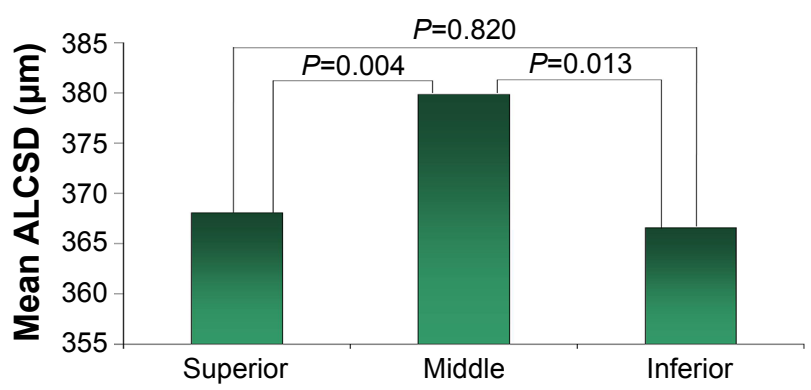

Figure 10 ALCSD variation at all planes.

Abbreviation: ALCSD, anterior lamina cribrosa surface depth.

groups were included. In addition, we did not demonstrate any correlation between ALCSD and IOP. Despite this, the presence of positive correlation between ALCSD and IOP level in glaucoma patients was supposed. ${ }^{13}$ This result may be because this study was conducted on only healthy subjects.

This study has the following limitations. All enrolled subjects were Saudi females; therefore, the results might not be totally applicable to males in Saudi Arabia and in other countries. So, further studies are recommended for males and for different ethnic populations to see the compatibility of the results. In addition, there were no different age groups. Moreover, the LC depth was measured at three points only in one scan line, decreasing the accuracy of the results. Furthermore, our study did not measure scleral thickness although many studies had confirmed its significant effect on ALCSD. This was because of the inability of our instrument to visualize the outer surface of the sclera. ${ }^{14}$ Additionally, the identification of the anterior LC surface was sometimes difficult in certain areas of the images due to the limitation of Topcon 3D OCT-2000.

\section{Conclusion}

This study is the first one to provide the normative profile of the ALCSD for Saudi females using Topcon 3D OCT-2000. Further studies are recommended for males, different ethnic populations, high myopic eyes, and different age groups using advanced imaging techniques such as enhanced depth imaging OCT.

Clinical Ophthalmology

\section{Publish your work in this journal}

Clinical Ophthalmology is an international, peer-reviewed journal covering all subspecialties within ophthalmology. Key topics include: Optometry; Visual science; Pharmacology and drug therapy in eye diseases; Basic Sciences; Primary and Secondary eye care; Patient Safety and Quality of Care Improvements. This journal is indexed on

\section{Acknowledgment}

This research project was supported by a grant from the Research Center of the Female Scientific and Medical Colleges, Deanship of Scientific Research, King Saud University.

\section{Disclosure}

The authors report no conflicts of interest in this work.

\section{References}

1. Park S, Ritch R. High resolution in vivo imaging of the lamina cribrosa. Saudi J Ophthalmol. 2011;25(4):363-372.

2. Crawford Downs J, Roberts MD, Sigal IA. Glaucomatous cupping of the lamina cribrosa: a review of the evidence for active progressive remodeling as a mechanism. Exp Eye Res. 2010;93(2):133-140.

3. Jonas JB, Berenshtein E, Holbach L. Lamina cribrosa thickness and spatial relationships between intraocular space and cerebrospinal fluid space in highly myopic eyes. Invest Ophthalmol Vis Sci. 2004;45(8):2660-2665.

4. Sigal I, Wang B, Strouthidis N, Akagi T, Girard M. Recent advances in OCT imaging of the lamina cribrosa. Br J Ophthalmol. 2014;98: ii34-ii39.

5. Seo JH, Kim TW, Weinreb RN. Lamina cribrosa depth in healthy eyes. Invest Ophthalmol Vis Sci. 2014;55(3):1241-1250.

6. Yang H, Downs JC, Girkin C, et al. 3-D histomorphometry of the normal and early glaucomatous monkey optic nerve head: lamina cribrosa and peripapillary scleral position and thickness. Invest Ophthalmol Vis Sci. 2007;48(10):4597-4607.

7. Strouthidis NG, Fortune B, Yang H, Sigal IA, Burgoyne CF. Longitudinal change detected by spectral domain optical coherence tomography in the optic nerve head and peripapillary retina in experimental glaucoma. Invest Ophthalmol Vis Sci. 2011;52(3):1206-1219.

8. Furlanetto RL, Park SC, Damle UJ, et al. Posterior displacement of the lamina cribrosa in glaucoma: in vivo interindividual and intereye comparisons. Invest Ophthalmol Vis Sci. 2013;54(7):4836-4842.

9. Sawada Y, Hangai M, Murata K, Ishikawa M, Yoshitomi T. Lamina cribrosa depth variation measured by spectral-domain optical coherence tomography within and between four glaucomatous optic disc phenotypes. Invest Ophthalmol Vis Sci. 2015;56(10):5777-5777.

10. Park SC, Brumm J, Furlanetto RL, et al. Lamina cribrosa depth in different stages of glaucoma. Invest Ophthalmol Vis Sci. 2015;56(3): 2059-2059.

11. Kim TW, Kim M, Weinreb RN, Woo SJ, Park KH, Hwang JM. Optic disc change with incipient myopia of childhood. Ophthalmology. 2012;119(1):21-26.

12. Ren R, Yang H, Gardiner SK, et al. Anterior lamina cribrosa surface depth, age, and visual field sensitivity in the Portland Progression Project. Invest Opthalmol Vis Sci. 2014;55(3):1531-1531.

13. Lee E, Kim T, Weinreb R, Kim H. Reversal of lamina cribrosa displacement after intraocular pressure reduction in open-angle glaucoma. Ophthalmology. 2013;120(3):553-559.

14. Bellezza AJ, Hart RT, Burgoyne CF. The optic nerve head as a biomechanical structure: initial finite element modeling. Invest Ophthalmol Vis Sci. 2000;41(10):2991-3000.
PubMed Central and CAS, and is the official journal of The Society of Clinical Ophthalmology (SCO). The manuscript management system is completely online and includes a very quick and fair peer-review system, which is all easy to use. Visit http://www.dovepress.com/ testimonials.php to read real quotes from published authors. 